\title{
Conservation Costs of Plant Genetic Resources for Food and Agriculture: Seed Genebanks
}

\author{
Anurudh K. Singh • K. S. Varaprasad • \\ Kamala Venkateswaran
}

Received: 12 January 2012/ Accepted: 19 July 2012/Published online: 15 August 2012

(C) NAAS (National Academy of Agricultural Sciences) 2012

\begin{abstract}
Recognizing the importance of plant genetic resources for food and agriculture and the sovereign rights conferred on the countries by the Convention on Biological Diversity, concerted efforts are under way worldwide toward the conservation of these priceless genetic resources. The information available on this topic largely dwells on the value and opportunity offered by such conservation, rather than focus on the costs of establishing the required facilities and or on the performance of the various activities necessary to meet the planned objectives. This study is based on the practical experience gained in the preparation of large collections and in the restoration of the collections of Indian origin from the International Crops Research Institute for the Semi-Arid Tropics, to the Indian National Program. In this study, the costs were calculated for the establishment of the facilities, acquisition of the germplasm, processing of the seed material, storage of the seed material as per the objectives in medium- or long-term stores, monitoring of germplasm to keep it dynamic, and regeneration/rejuvenation of accessions falling below genebank standards in crops with different breeding systems, different seed sizes and compositions. The article draws inferences about the financial commitment needed and future conservation strategies for formulating cost-effective conservation approaches.
\end{abstract}

Keywords Germplasm acquisition - Germplasm monitoring - Germplasm regeneration - Plant genetic resources . Seed processing $\cdot$ Seed storage

\section{Introduction}

With increased awareness of the value of plant genetic resources for food and agriculture (PGRFA), conservationrelated activities have increased worldwide, and the cost of such activities has received much attention. Several studies have been carried out using different methodologies $[2,4$,

\footnotetext{
A. K. Singh $(\bowtie)$

Department of Genetics, M.D. University, Rohtak 124 001, India e-mail: anurudhksingh@gmail.com

K. S. Varaprasad

Directorate of Oilseeds Research, Rajendranagar,

Hyderabad 100 009, India

K. Venkateswaran

National Bureau of Plant Genetic Resources, Regional Station, Hyderabad 500 030, India
}

7-9] and debated [5, 6]. However, except for Pardey et al. [8], most studies have only concentrated on discussing the gains and opportunities offered by the conservation of genetic resources. On the other hand, a study that focuses on the cost of conservation (which is directly related to the desired objectives and crops to be conserved) would go a long way in improving the effectiveness of conservation measures and in answering some of the key conservation management questions, such as: What resources should be conserved? How should they be conserved? Where should they be conserved? When should they be regenerated? How should they be used/rationalized to make conservation cost-effective? A cost-of-conservation study would also draw inferences that could guide future conservation strategies and help in formulating cost-effective conservation approaches. Conservation is a key activity in the postConvention on Biological Diversity (CBD) era, and the numbers of stakeholders have dramatically increased in 
Table 1 Candidate crops selected and their features

\begin{tabular}{|c|c|c|c|c|}
\hline Pollination mechanism & Seed size & Oily/non-oily & Candidate crops & Additional features \\
\hline \multirow[t]{4}{*}{ Self-pollinated } & \multirow[t]{2}{*}{ Small seed } & Non-oily & Wheat and rice & $\begin{array}{l}\text { High multiplication rate, storage of cultivated species seeds is } \\
\text { simple, storage of wild relatives, and inter-specific hybrids } \\
\text { may pose problems }\end{array}$ \\
\hline & & Oily & Sesame & High multiplication rate \\
\hline & \multirow[t]{2}{*}{ Medium-large seed } & Non-oily & Chickpea & Moderate multiplication rate \\
\hline & & Oily & Groundnut & Autogamous, low multiplication rate \\
\hline \multirow[t]{4}{*}{ Cross-pollinated } & \multirow[t]{2}{*}{ Small seed } & Non-oily & Pearl millet & High multiplication rate \\
\hline & & Oily & Mustard & $\begin{array}{l}\text { Wind pollinated, sporophytic incompatibility, high } \\
\text { multiplication rate }\end{array}$ \\
\hline & \multirow[t]{2}{*}{ Medium-large seed } & Non-oily & Maize & Medium seed multiplication rate \\
\hline & & Oily & Castor & Obligate cross-pollinated species, unisexual flowers \\
\hline \multirow[t]{3}{*}{ Often cross-pollinated } & Small seed & Non-oily & Sorghum & $\begin{array}{l}\text { High seed multiplication rate, significant variation in seed } \\
\text { color }\end{array}$ \\
\hline & \multirow[t]{2}{*}{ Medium-large seed } & Non-oily & Pigeonpea & Semi-perennial, variation in seed size, insect-pollinated \\
\hline & & Oily & Cotton & Semi-perennial, a poor storer \\
\hline
\end{tabular}

view of the shift of sovereign rights to the countries possessing the genetic resources or germplasm. Several developing nations are planning to establish and develop their own genebanks. At the same time, civil societies are planning to facilitate farmers' requirements, and private firms are increasingly eyeing germplasm resources, especially forest genetic resources. Today, there are some 1,750 individual genebanks worldwide with total holdings of about 7.4 million accessions, of which about 6.6 million are held in national genebanks [3]. In India alone, the National Genebank (NGB) holds around 0.39 million accessions as its base collection [13], and nearly equal numbers are being held by 41 National Active Germplasm Sites (NAGS) spread over major agro-climatic zones [10].

The assessment of the cost of conservation is particularly useful for developing countries: it facilitates the objective allocation of financial assistance and enables the calculation of the overall economic benefits that can be drawn from germplasm conservation. Conservation of PGRFA contributes to food, nutrition, and health security, and makes available sources of genes that may help in increasing productivity and in overcoming yield-reducing factors, thereby facilitating sustainable agriculture. Cost estimation helps to identify the requirements for conservation of PGRFA and to determine the priorities, as many of the resources might not have immediate applied value and might not be conserved by farmers in situ, while nevertheless possessing scientific value. The overall cost can be reflected in terms of the cost: benefit ratio (i.e., the fiscal input required and the opportunity offered), though the cost:benefit ratio is difficult to quantify in view of the long gestation period involved. The running costs represent the budget required for establishing and running the ex situ conservation facilities, and associated activities, to determine step-wise monetary expenses. They would include hidden costs such as depreciation costs, institutional costs, and the costs incurred in follow-up of national legislations and regulations for ex situ conservation of PGRFA. In addition, there could be some non-fixed costs, such as compensation costs paid to farmers during collection or for acquisition of the material. The opportunity cost would be the visualization of benefits that a country could harness by conserving genetic diversity, which may become the basis for increasing genetic potential to improve the physical and nutritional quality and productivity (national production) of crops and/or overcome a yield-reducing factor, thus helping recover yield losses.

This study takes into consideration the capital cost invested to establish the conservation facilities and the cost of the basic activities performed for ex situ conservation of collections as seed, by storage, the most commonly used and economically less demanding method for conservation of genetic resources, in crops genetically diverse in their breeding system, seed size and composition. The study therefore includes the establishment cost, the cost of collection or acquisition of germplasm, evaluation of genetic and physical purity and quality of seed, monitoring of seed viability through germination tests, seed quantity and health, seed processing as per established genebank standards, seed storage, and regeneration of accessions falling below standards and data management. It does not include the cost of characterization, evaluation, and distribution of the seed for use and/or restoration of collections.

The analyses presented here are based on the experience gained at the National Bureau of Plant Genetic Resources 
(NBPGR, India) during the collection missions, and restoration of Indian collections from International Crops Research Institute for Semi-Arid Tropics (ICRISAT, Patancheru, India). The Indian National Genebank (NGB) established at NBPGR has 12 long-term store (LTS) modules maintained at a temperature of $-18{ }^{\circ} \mathrm{C}$, and one medium-term store (MTS) module maintained at a temperature of $4-7{ }^{\circ} \mathrm{C}$ at $35-40 \%$ relative humidity (RH). The collections are conserved under a network approach, with the consolidated long-term storage at NGB, and dispersed crop-wise medium-term storage in facilities at the NAGS located at crop-based institutes (under universities, or the Indian Council of Agricultural Research, ICAR) and seven NBPGR Regional Stations located in different bio-geographic regions. The NGB is responsible for long-term conservation and to facilitate the activity of the NAGS, with need-based restoration and enrichment of active collections. The NAGS are responsible for further evaluation, multiplication, regeneration, and distribution of germplasm. Several of them have MTS facilities maintained at a temperature of $4-7{ }^{\circ} \mathrm{C}$ at $35-40 \% \mathrm{RH}$.

The sample size has a significant implication on cost and management. For long-term conservation, the size of the seed sample is 2,000 seeds for self-pollinated crops and 5,000 seeds for cross-pollinated crops. For medium-term conservation, $250-1,000 \mathrm{~g}$ seed material is stored depending on the seed size and the demand for use in research and crop improvement. For long-term storage, the accessions are packed in aluminum-laminated pouches of appropriate size, whereas for medium-term storage, they are packed and stored in various types of moisture-proof containers. The cost of each of the items has been estimated in US dollars, applying an exchange rate of US $\$ 1=$ Indian Rs. 46. The costing in this study is based on the prices/tariffs prevailing in 2004-2005. For example, during this period, the local electricity tariff was $\$ 0.1$ per unit; the average price of diesel was $\$ 0.57$ per liter; the daily wages for labor were $\$ 2.33$ (semi-skilled) and $\$ 2.5$ (skilled). As for equipment, a seed germinator costed $\$ 1087$, while a seed drier could be bought for $\$ 10,869$.

\section{Materials and Methods}

This study attempts to identify the various essential cost components associated with different activities for efficient ex situ conservation of germplasm as seed. To cover the total spectrum of variability in breeding behavior, multiplication rate, physical seed size, and biochemical composition, the candidate crops included self-pollinated, cross-pollinated, and often cross-pollinated crops; large-, medium-, and small-seeded crops; and oily and non-oily seed crops with diverse multiplication rates.

\section{Candidate Crops}

The choice of candidate crops-wheat, rice, sesame, chickpea, groundnut, pearl millet, mustard, maize, castor, sorghum, pigeonpea, and cotton-was governed by the need to observe a comprehensive range of variables that could influence the cost of storage of seeds of different crop species (Table 1).

\section{Methodology of Costing}

For the purpose of estimation, the costs have been categorized into capital cost, collection/acquisition cost, seed processing cost, storage cost, maintenance/monitoring cost, and regeneration cost. They can also be classified along three major variables: fixed costs (capital needed to establish facilities, including equipment); semi-fixed costs (collection, processing, production/multiplication, and monitoring and regeneration costs); and hidden costs (seed dormancy breaking and variable marginal costs, such as compensation during acquisition, etc.). In addition, the study took into account some common costs that are constant, such as those related to capital input incurred in the establishment of basic infrastructure, salaries of core staff, cost of human resource development, and administrative and institutional charges. Thus, in order to arrive at an average per accession cost, it was necessary to consider all the candidate-crop accessions conserved during the entire study period. In the present case, this was from early 2000 to 2005 , a phase that saw a rapid increase in the number of accessions, consequent to the collections made under the World Bank funded National Agricultural Technology Project, and restoration of Indian germplasm from ICRISAT.

As mentioned earlier, the costing in this study is based on the prevailing costs of the various items during 2004-2005, costs that increased substantially in subsequent years. For example, between 2004/2005 and 2010/2011, the electricity tariff rose from $\$ 0.10$ to 0.14 per unit; the price of diesel increased from $\$ 0.57$ to 0.71 per liter; and the daily labor wage went up from $\$ 2.30$ to 2.50 (semiskilled) and from $\$ 6.20$ to 6.80 (skilled). This underlines the importance of taking into account the inflation rate, which would vary from country to country, and year to year. For example, in India, between the years 1969 and 2010, the average inflation rate was $7.99 \%$; it increased further to $9.72 \%$ by September 2011 [1]. Therefore, for a more accurate picture of the costs and the expected escalation, the annual inflation rate must be incorporated in the costing for the reference period.

The following sections offer an overview/description of the different categories of costs adopted by this study, and which are typically applicable in the setting up of any seed 
bank. On the other hand, the actual costs incurred in the framework of the study are presented in the various tables, and analyzed in the "Results and Discussion" section.

\section{Capital Costs}

The capital costs are those related to the establishment of facilities, and for equipment required for seed processing, storage, regeneration, health test, information documentation, etc. (Table 2). In addition, they would include the costs of repair and/or replacement of either the equipment or its components, which are covered under the overheads. This expenditure is not directly related to the annual cost for an accession; however, if divided by the holding or processing capacity of 25,000 accessions for a period 25 years, it would reflect one-time expenses on an accession. For exact calculation, it may include the annual depreciation (1-5\%) from purchase price, service life and rate of interest on the capital. Table 2 lists the capital items, equipment, and facilities based on expenses made by the NGB or on the current replacement cost. The presumed costs for processing and storage of the candidate-crop seed material (25-35 years for MTS, and 35-50 years for LTS) have been calculated with variation in capacity of stores and equipments. Therefore, if seed size is taken into account for a holding of 25,000 accessions, the storage space required would vary, but the operation cost may be constant. This is because the costs of staff and labor would remain the same irrespective of the crops stored, the pattern of use of the established facilities and the maximum holding capacity.

\section{Germplasm Acquisition Costs}

During exploration, emphasis is laid on the collection of local landraces, farmers' varieties from farmers' fields and wild relatives from remote or interior regions. Broadly, two kinds of collections are made: crop- and region-specific collections. Sometimes, special missions are carried out for the collection of threatened diversity or trait-specific diversity under a targeted collection. The duration of the trip and number of accessions collected depend on the distribution and availability of the germplasm. In general, the collection expeditions for orthodox seed species are longer, with more accessions collected than in the case of "recalcitrant" or "vegetatively propagated" crops. The acquisition costs are calculated on the basis of the number of samples collected in a specific crop in a given number of days. The local topography and access to the target region can significantly influence the acquisition cost.

By its very nature, collection is time intensive: a large number of staff-hours are spent on recording of the passport data, that include information on habitat, nature of plants, growth, socio-economic value, ethno-botanical aspects, reaction to prevailing biotic and abiotic stresses, role of various tribes or ethnic groups and women, etc. Collection may involve the use of certain sophisticated equipment, such as geographical positioning system (GPS) devices, in addition to routine equipment. The cost of augmentation/collection (genetic diversity) may include various hidden costs, such as those for pre-surveys, coordination with collaborators, developing formats for recording of passport information, guides, manpower, etc. Commercial crops such as groundnut may involve further hidden costs, such as compensatory payments to farmers for sharing of their germplasm.

Acquisition of germplasm involves transportation costs. In addition, import of germplasm implies costs for meeting various legislative requirements, such as import permits, phytosanitary certificates, quarantine, safety requirements, etc. The per accession cost can be easily obtained by dividing the total cost by the number of accessions received. Introductions may have an additional component for multiplication, based on the number of seeds supplied, the breeding system of the crop, multiplication rate, etc. Acquisition costs can be typically high in crops such as groundnut: the number of seeds supplied and multiplication rate is usually low, and indexing becomes imperative to restrict the entry of seed-borne viruses.

\section{Seed Processing Costs}

Once received at the genebank, the seed material must be checked against the list provided by the donor/collector, in order to verify the material's identity. The physical verification of the material and the selection of physiologically mature and healthy seed may sometimes require special methods/equipment, which invariably increases the cost: sorting by density gradient, cleaning of seed to ensure storage of high-quality seed, removal of undesirable types (variants, broken, and diseased seeds), etc. This activity may be comparatively easier in self-pollinated crops such as wheat or rice, but requires far greater attention in crosspollinated or often cross-pollinated crops such as maize, pearl millet, pigeonpea, etc. Further, a germination test is conducted to ensure conservation of quality seed having the desirable viability ( $>65$ and $85 \%$, in the case of active/ base collections for medium- and long-term storage, respectively). The germination test is a time- and laborintensive and expensive activity. To meet the established standards, the material needs to be dried to a prescribed moisture level: $8-10 \%$ for medium-term and 3-7 \% for long-term storage. The costs for performing these activities include the maintenance of equipment, seed germination test, and seed drying at the time of entry along with the labor cost. The processed seed subsequently needs to be 


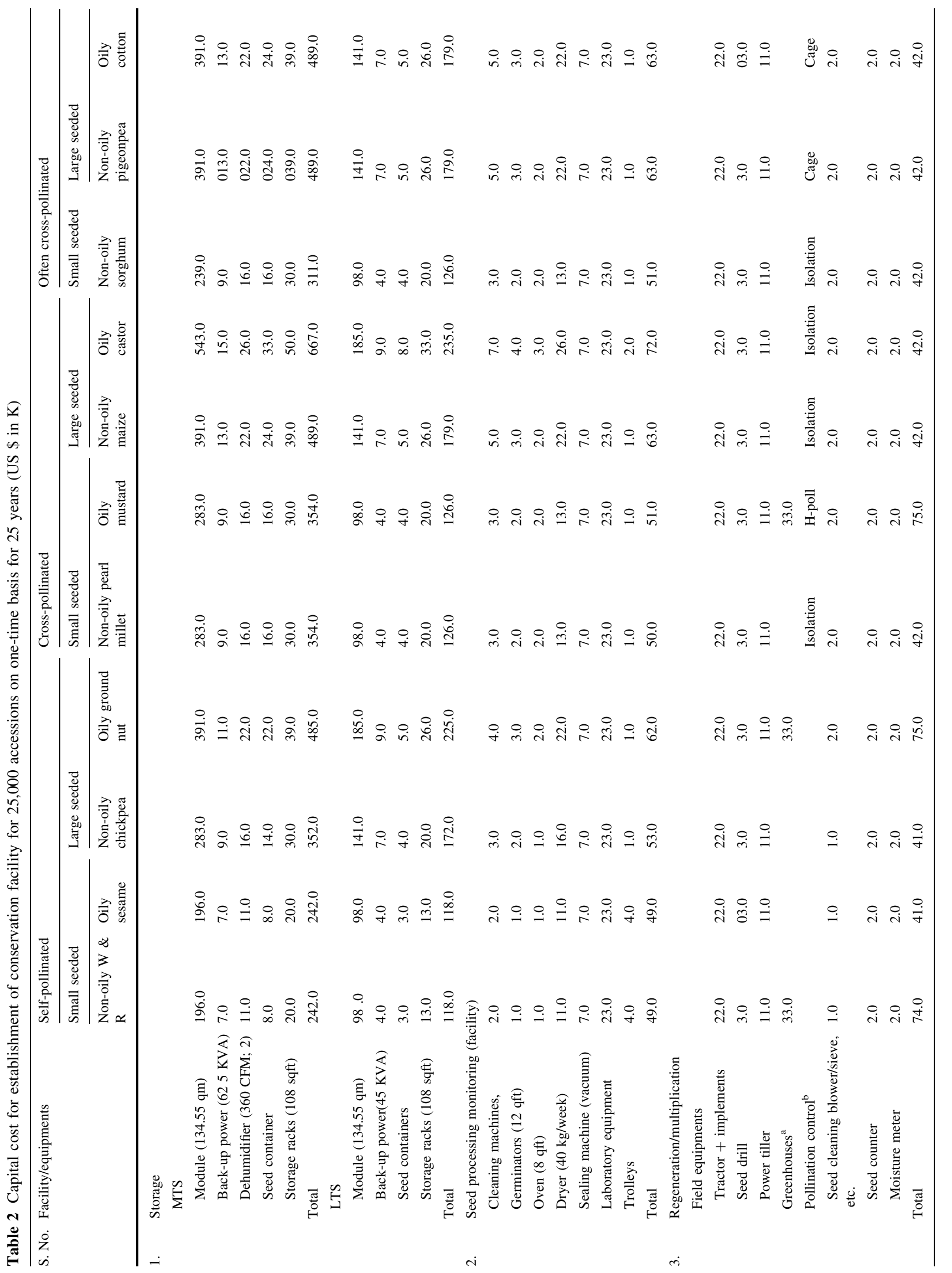




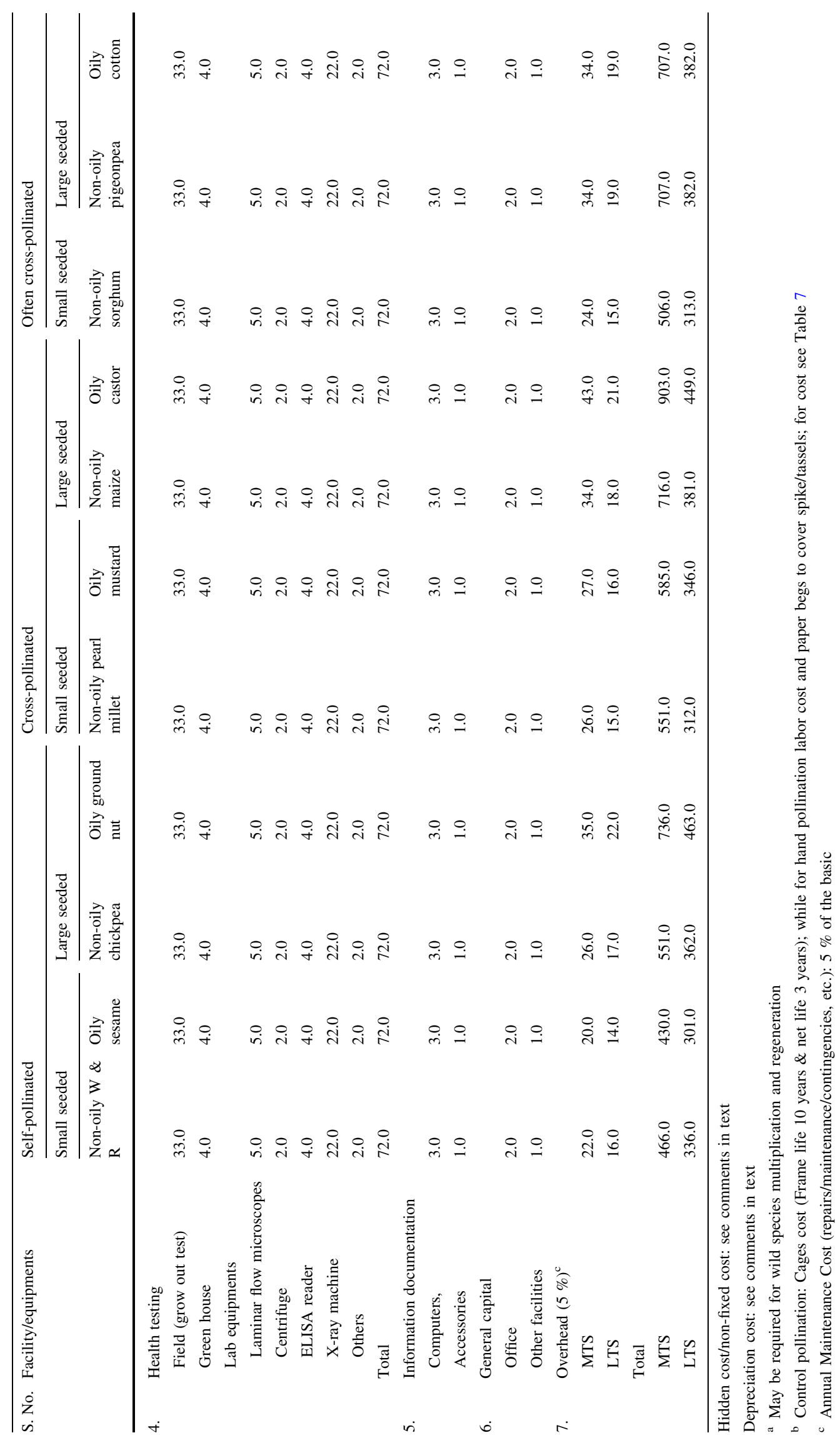


packaged in appropriate containers. The passport information for each accession, logged at the time of collection or supplied during introduction, has to be tabulated and entered into suitable databases for information management and to facilitate various analyses, monitoring, and retrieval of material.

\section{Storage Costs}

Any controlled-environment storage facility is a costly affair, but the costs of ensuring germplasm storage-under precise control of low temperatures and low $\mathrm{RH}-$ are highly variable particularly in tropical countries such as India. Seasonal variations contribute to increase running costs of various equipments due to higher power consumption, with additional labor costs for monitoring and maintenance, and to ensure uninterrupted power supply through standby generators. Costing for these operations in a multiple-crop genebank is difficult. However, because the costs are related to physical operations and maintenance of the facility, they may be considered constant. The costing presented in this study has been made based on energy used for maintenance and operation of the genebank on an annual basis, without accounting for climatic variations. Similarly, although the cost of routine climate control, organization, and monitoring may vary, it has been considered constant as it involves core staff on a regular payroll, while the cost of maintenance of equipment (including the generator) has been covered under the overheads.

The seed material is packed in laminated aluminum pouches for long-term storage, and in moisture-proof plastic, glass or aluminum bottles for medium-term storage. Table 2 gives the costs of various types of containers of different sizes suited to various crops. The other essential cost is for the management of information regarding the stored accessions; this involves the costs for the creation, update, and management of databases dealing with passport and genebank management data. Information management costs include the salaries of data entry operators. Other general management costs include the hidden cost of genebank curators, technical staff, and other common operations. As such, expenses represent a bulk cost related to the common functions performed during conservation, they too are considered constant.

\section{Monitoring Costs}

Stored seed undergoes gradual deterioration over time due to aging, losing viability. Thus, loss in seed viability is an indicator of possible genetic changes affecting genetic integrity. To ensure conservation of high-quality seed, the seed viability (through germination tests), seed health (checks for presence of pathogens/pests), and seed quantity (seed count against database records) are usually monitored at intervals of 5 years for active collections, and 10 years for base collections. The same standards are followed as those applicable for introduction of accessions. Drop in seed number, viability or poor health would necessitate regeneration. The labor costs for assessment and for running the laboratory and equipment such as germination chambers, etc., constitute a large chunk of the monitoring costs. The costs incurred by the various components of the monitoring activity are included only for the year of monitoring. The cost of maintenance of related equipment is covered under the overheads.

\section{Regeneration Costs}

The accessions identified for regeneration are retrieved in requisite quantities as per the genetic structure of the crop in question. The population raised should capture the total spectrum of genetic and allelic variability of that crop. As all the crops cannot be regenerated at the site of a multicrop genebank, seed of certain crops will need to be dispatched to appropriate location (e.g., NAGS in the crop cultivation area) for regeneration/multiplication. The regeneration of self-pollinated crops such as wheat and rice is simple, and may only need small populations, and could be managed even in screen houses. However, some crops would need special arrangements to meet the ecological requirements and multiplication rates for the generation of truthful seed in sufficient quantities for conservation in MTS/LTS.

The amount of inputs, such as field preparation, irrigation, agro-chemicals, and crop management varies according to the crop, season, and other factors. For field regeneration, disease-free zones or post-rainy seasons are more suitable. Harvesting of crop may require different inputs: for example, groundnut, in addition to its low multiplication rate, has large and food-rich seed that would require manual harvesting and threshing, which increases the cost of regeneration. In the present analysis, the cost of regeneration for an accession has been included only for the year in which that accession was regenerated.

\section{Results and Discussion}

The detailed component-wise expenditure-incurred in the specific context of this study-for the establishment of conservation facility has been tabulated in Tables 2, 3, 4, 5, 6 , and 7. Table 8 presents the consolidated information, giving overall expenditure toward capital cost and for each of the activities. The first column of this table presents the major activities. In addition, costs for some activities that 


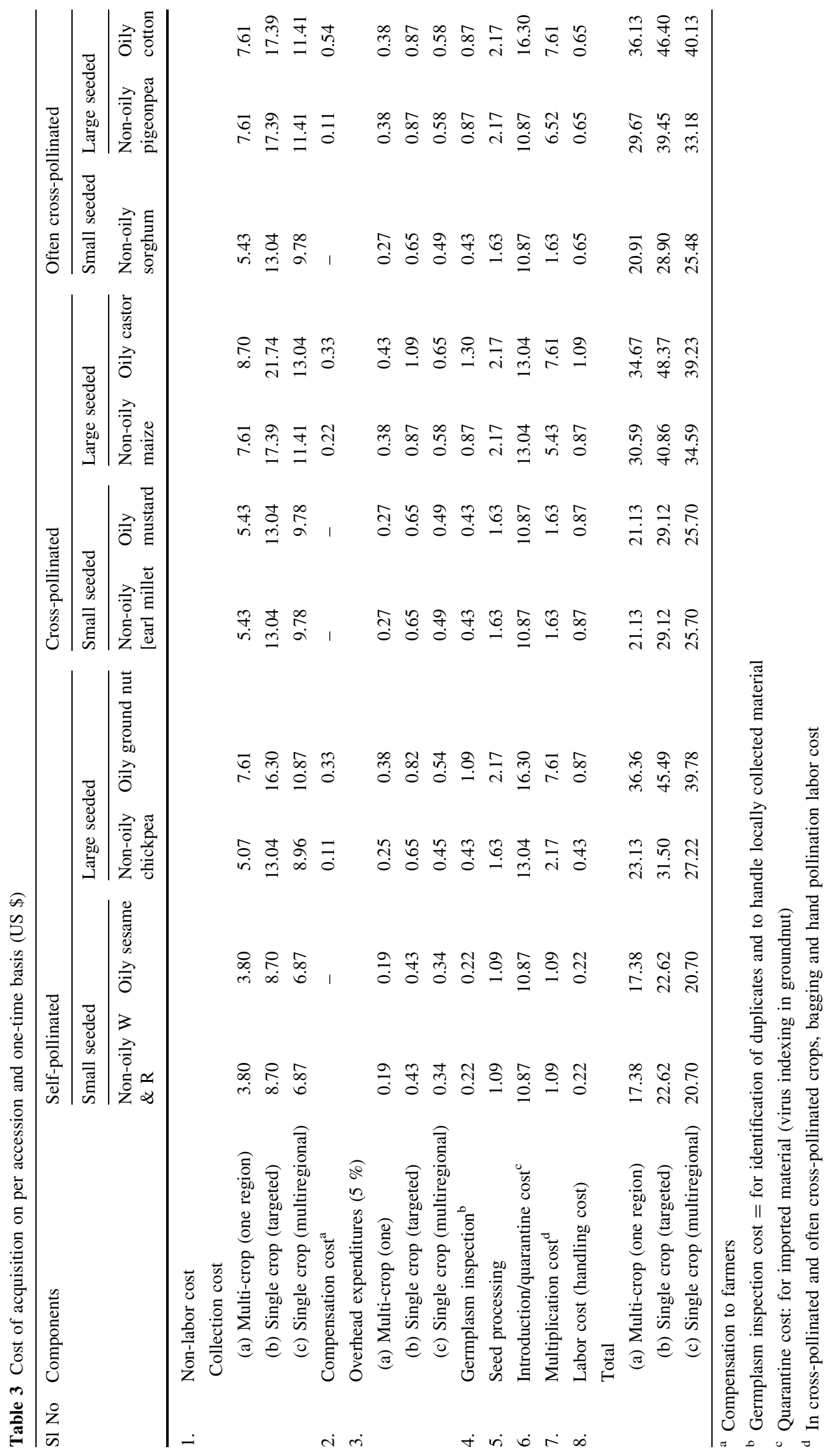




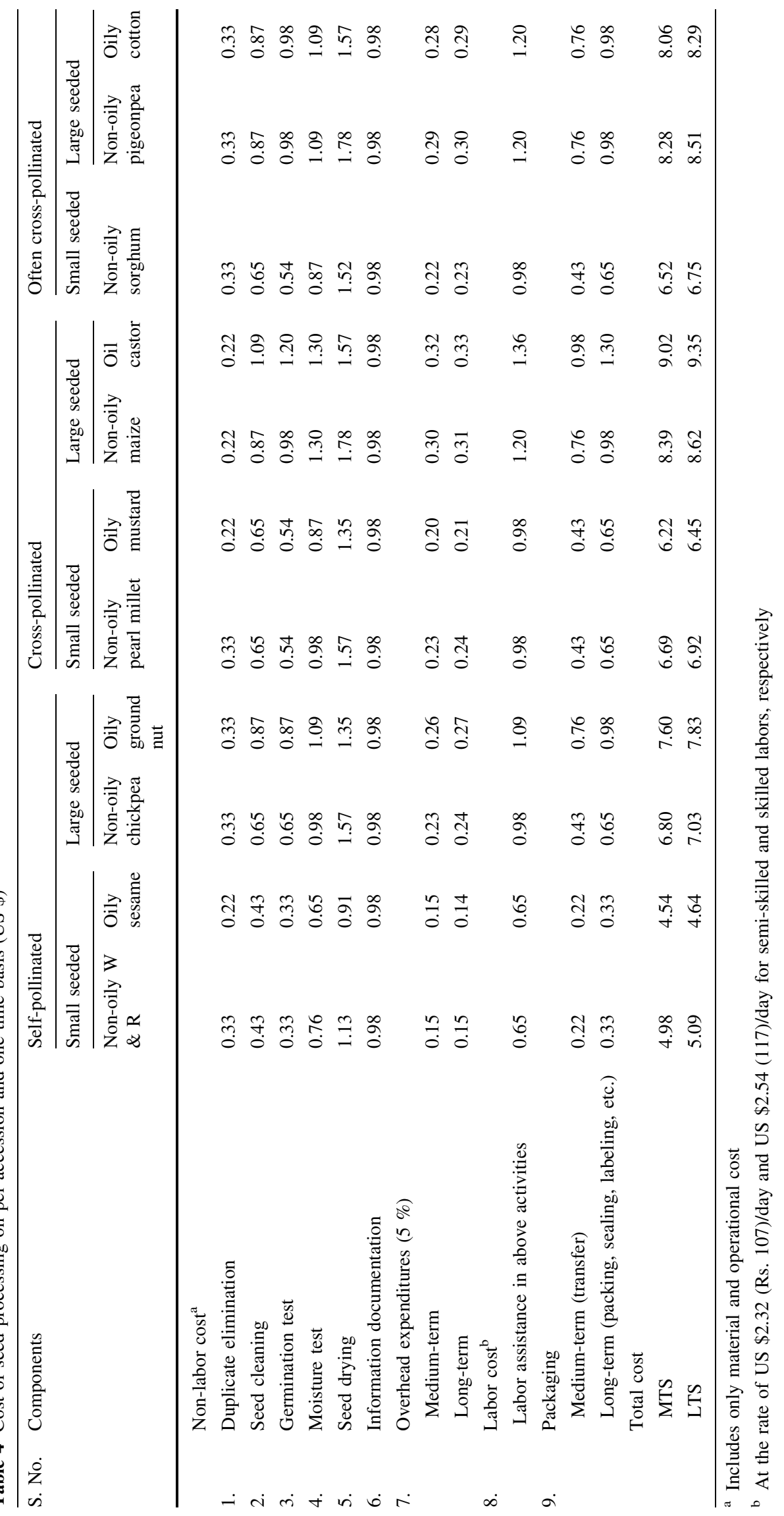




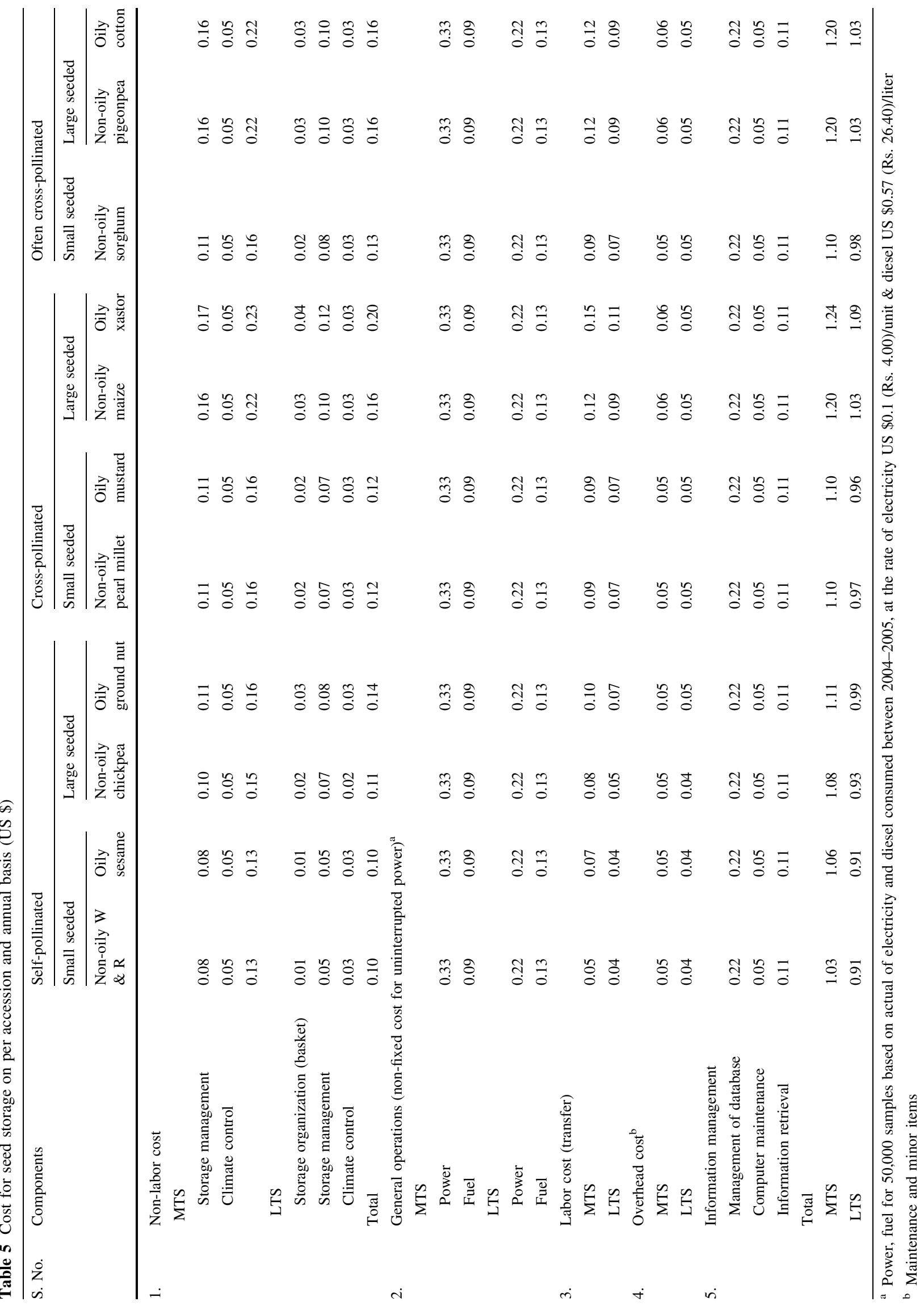




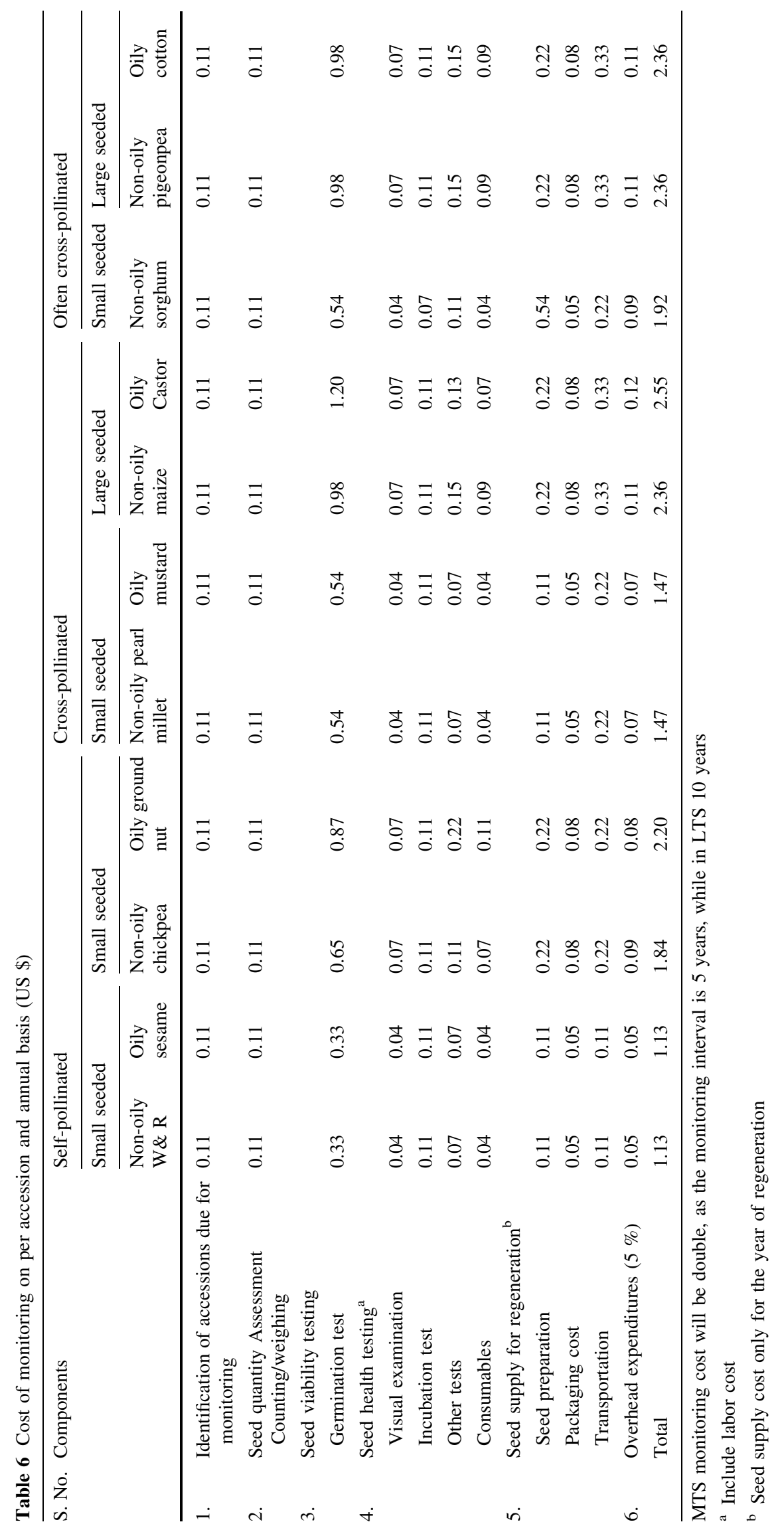




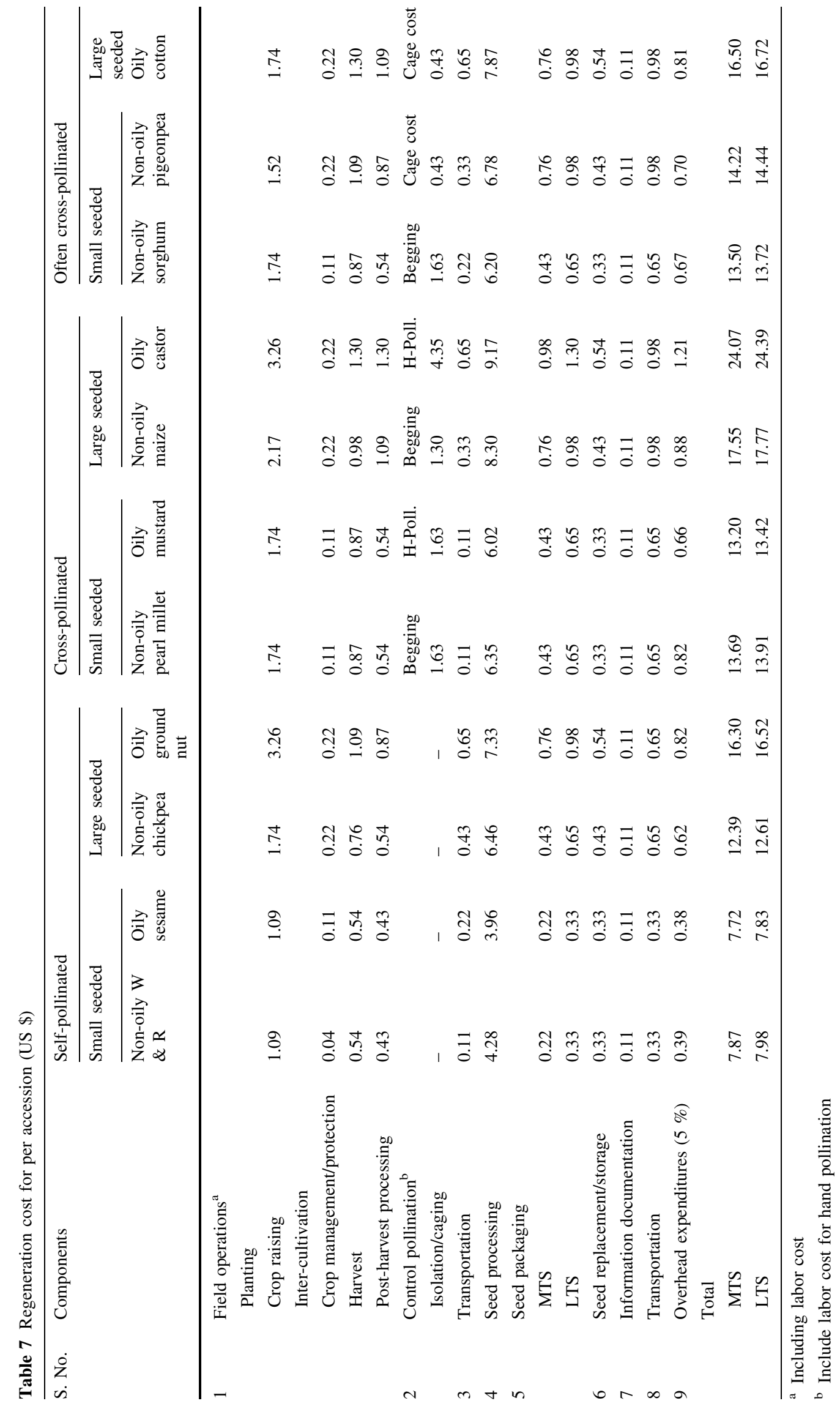




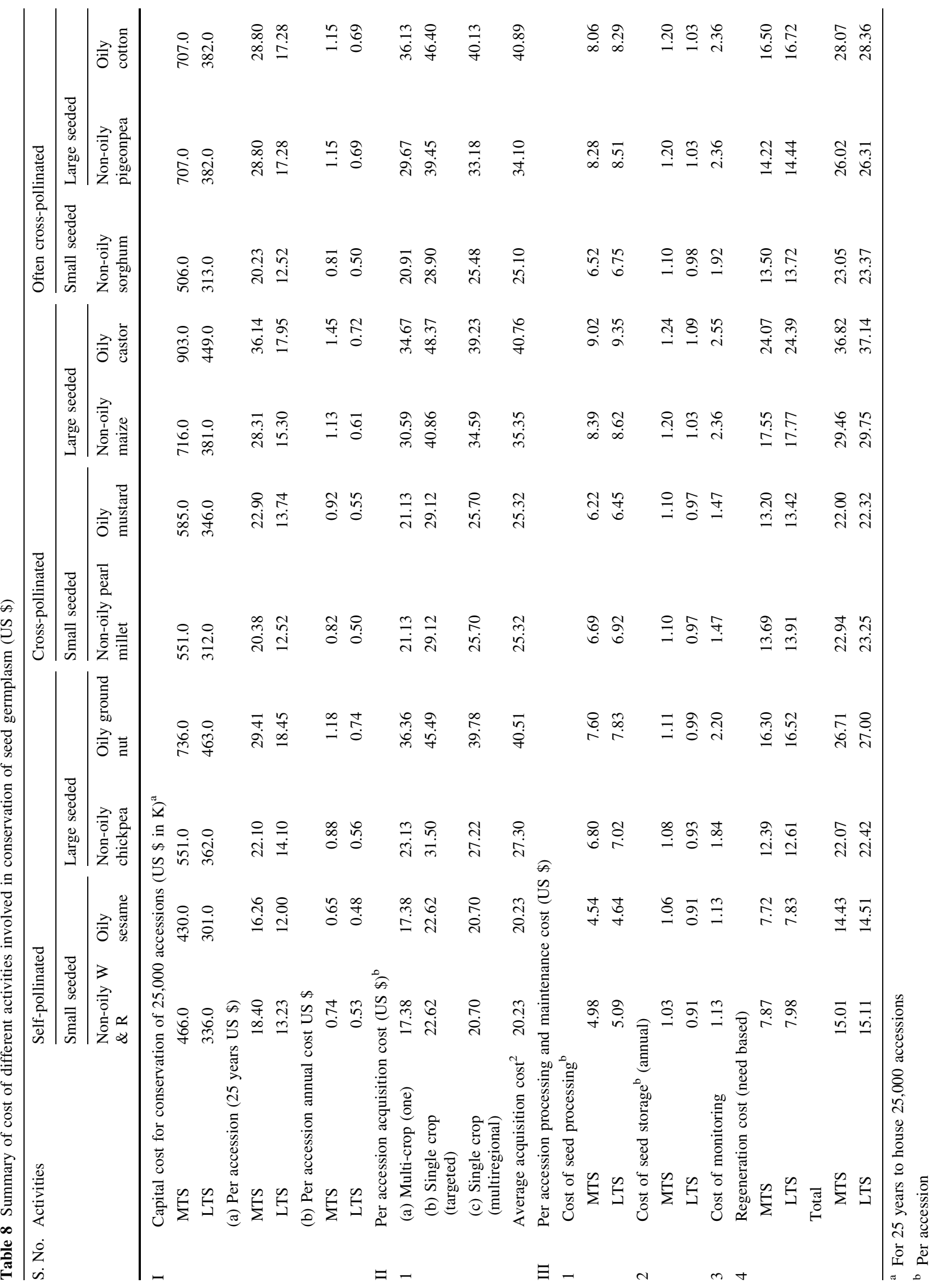


were common across crops and needed core technical and managerial staffs have been considered essential and constant. On the other hand, some specific crops needed nonroutine activities, such as breaking of seed dormancy in wild species and regeneration of crops for base collections for posterity and safety. In this study, accessions of most ICRISAT mandate crops were regenerated at relevant sites in collaboration with the respective All-India Coordinated Research Projects and then transported to the National Genebank, NBPGR at New Delhi, contributing to an increase in the costs. This kind of a situation may upset the contemporary average cost calculated on a per year basis in relation to contemporary crops/accessions and conditions. However, a typical set of accessions, which passed through all the steps has been used in this study to calculate the capital cost and cost on a per accession basis for a more representative estimate of each activity.

For the same space, the establishment cost was higher for an MTS facility than for an LTS facility (Table 2). This is because of the additional requirement of a dehumidifier in the MTS for precise control of the RH. This component also pushes up the energy and maintenance costs of MTS facilities, particularly in tropical countries like India, where seasonal variations cause fluctuations in $\mathrm{RH}$, leading to more intense use of dehumidifiers to keep the RH within the recommended limits. Another major factor influencing the establishment cost is the seed size of the mandate crop: larger the seed size, higher the establishment cost, because fewer numbers of accessions can be accommodated in the limited space of the controlled-environment facility. The

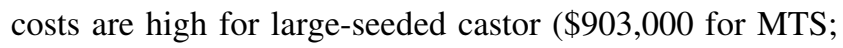
$\$ 449,000$ for LTS) and groundnut (\$736,000 for MTS; $\$ 463,000$ for LTS), followed by medium-seeded crosspollinated maize and often cross-pollinated pigeonpea and cotton. These findings corroborate the earlier observations reported by Pardey et al. [8] and Koo et al. [6]. Smallseeded self-pollinated oilseed sesame (\$430,000 for MTS; $\$ 301,000$ for LTS) and non-oil rice and wheat $(\$ 466,000$ for MTS; $\$ 336,000$ for LTS) have the lowest capital costs (Table 2). In 2008 (reference year), the International Institute for Tropical Agriculture (IITA, Nigeria) spent $\$ 358,143$ and $\$ 28,217$ annually on the conservation and management of cowpea and wild Vignas. The capital costs took the major share of the costs. Medium-seeded cowpea cost about $\$ 72$ per accession, and small-seeded wild Vigna only cost about half as much [14].

In general, the cost of acquiring accessions through collection was the highest in instances of targeted collections of a specific species, compared with multi-crop collection from a single region, or a single-crop collection from diverse regions (Table 3). Again, the per accession acquisition costs were highest for large-seeded, bulky oilseed crops such as castor (\$34.67-48.37) and groundnut
(\$36.36-45.49), followed by the medium-seeded cross- or often cross-pollinated crops such as maize, cotton, and pigeonpea (Table 3 ). The acquisition costs were minimal for small-seeded self-pollinated crops such as oily sesame and non-oily wheat and rice (\$17.38-22.62, per accession). The experience may reflect the approximate number of accessions that could be collected during a trip in $n$ number of days, and the number of explorations and the duration of explorations required for collection of targeted collections. The quarantine cost for introductions, although uniform, was higher in crops such as groundnut, which needed additional tests, for indexing for seed-borne viruses, low multiplication rate, etc.

Table 4 lists the non-labor and labor costs for processing of the seed material in candidate crops starting from duplicate elimination, cleaning, etc. Again, the cost of seed processing was highest for the large-seeded oil cropscastor (\$9.02 for MTS; \$9.35 for LTS) and groundnut (\$7.60 for MTS; $\$ 7.83$ for LTS)_followed by the medium-seeded cross-pollinated maize and often cross-pollinated cotton and pigeonpea (Table 4). The processing costs were lowest for self-pollinated small-seeded oil crop sesame (\$4.64 for LTS; \$4.54 for MTS) and non-oil crops rice/wheat ( $\$ 5.09$ for LTS; $\$ 4.98$ for MTS). The seed processing costs were higher for LTS (seeds needed to be dried to a lower moisture level) than for MTS. For longterm conservation, the seeds are packed in laminated aluminum pouches with hermetical vacuum sealing and labeling of each packet, while for medium-term conservation, comparatively larger quantities of seed are stored in moisture-proof reusable containers. Both are labor intensive. Table 2 presents the costs of containers.

The cost of seed storage was highest for large- and medium-seeded cross-pollinated castor and maize $(\$ 1.24$ for MTS; \$1.09 for LTS) and often cross-pollinated pigeonpea and cotton (\$1.20 for MTS; \$1.03 for LTS) (Table 5). Seed storage costs were lowest for small-seeded oily sesame (\$1.06 for MTS; \$0.91 for LTS) and for smallseeded non-oily wheat/rice (\$1.03 for MTS; \$0.91 for LTS), which corroborated earlier results $[6,8]$. Koo et al. [6] reported that holding seed samples for 1 year cost less than $\$ 1.5$ per accession for most crops, except for maize, which cost $\$ 2.16$. The costs for seed storage in MTS are higher than for storage in LTS, probably because the LTS is less frequently opened than MTS, which limits cooling losses, and thereby reduces the power consumption for running of compressors to maintain the desired temperatures. Further, in MTS, the operation of dehumidifiers for maintaining the recommended $\mathrm{RH}$ needs greater energy, proportionally increasing the operation cost. Other costs are nearly similar, except that germplasm proposed for MTS would need processing in larger quantities, and require greater power and fuel for uninterrupted power 
supply to facilitate the maintenance of desired temperature and RH. While this activity demanded the maximum workhours of the core technical and managerial staffs, it is uniform irrespective of the crops, and therefore considered constant. The transfer and monitoring of the stored accessions are seasonal activities requiring additional labor, which was again higher for large- and medium-seeded crops than for the small-seeded types (Table 5). Information management on the stored accessions is another important component to facilitate timely monitoring, regeneration, and distribution.

To keep the collections dynamic with the highest quality standards, each accession is monitored for the requisite number, recommended viability, and seed health at regular intervals. The expenses for this activity are nearly uniform, and the major deviation is caused by the expenses in testing of seed viability during the year of monitoring and supply of seed, if regeneration is needed. Regeneration cost is accounted only for the year of regeneration. This is higher again for large- and medium-seeded crops for the same reasons as for seed processing at the time of entry (Table 6). Therefore, cross-pollinated large-seeded castor (\$2.55) and maize (\$2.36), and medium-seeded often crosspollinated pigeonpea and cotton (\$2.36) had the highest monitoring cost, and the small-seeded self-pollinated wheat/rice, and sesame had the lowest cost (\$1.13) (Table 6). The monitoring costs would be higher for accessions in MTS than in LTS, because of the double frequency of monitoring.

In regeneration of accessions, the costs (\$for MTS; LTS) were considerably high in most of the cross-pollinated, often cross-pollinated, and self-pollinated crops with largeand medium-sized seed and low multiplication rate, such as castor $(24.07$; 24.39), maize $(17.55 ; 17.77)$, cotton (16.50; $16.72)$, pigeonpea $(14.22 ; 14.44)$, and groundnut (16.30; 16.52), which corroborated earlier observations [6, 8]. Crops such as cotton have high regeneration costs because of inherent issues such as perenniality, which contribute to diverse physiological maturity of seed at harvest resulting in poor storability. These differences need greater manual labor for handling of accessions thereby increasing the labor cost. In cross-pollinated crops, the population size required for capturing the total spectrum of genetic diversity is higher in addition to the usage of controlled pollination to restrict the genetic contamination through gene flow (Table 7). Further, Koo et al. [6] reported that regeneration costs for forage crops at International Center for Tropical Agriculture (CIAT, Cali, Colombia) and for wild rice accessions at International Rice Research Institute (IRRI, Los Baños, Philippines) are higher than those for chickpea and sorghum at ICRISAT, because of the higher cost of repeated regeneration.
Table 8 provides a summary of the total expenses, which have been divided into three major components: capital costs for establishment, costs for acquisition of collections, and costs for processing and maintenance. On a per accession basis, the cost for establishment of facilities for conserving an accession for 25 years is around $30-35 \%$ of the total annual cost incurred for MTS conservation, and around $25-30 \%$ of the total annual cost incurred for LTS conservation (Table 8). The establishment cost for each accession was calculated by dividing the total cost of the establishment by 25,000 accessions with a life-time of 25 years for the facilities. It can be further divided by 25 to calculate the annual cost. The annual storage costs for different crops ranged from $\$ 0.91$ to 1.24 (Table 8 ), similar to the cost of $\$ 1.0$ estimated by Walters [12] for storing soybean at $6 \%$ moisture and $-15^{\circ} \mathrm{C}$ per year over a period of 100 years. The other aspects discerned by the comparative analysis were that establishment cost and operation costs are lower for long-term conservation facilities than for medium-term conservation facilities. This is primarily because of the requirement of additional equipment to support maintenance of $\mathrm{RH}$ in the cold chambers. In operational costs, the processing and regeneration are expensive when an accession is prepared for long-term conservation, but storage is cheaper because LTS facilities are opened less frequently.

\section{Running Costs}

In addition to acquisition, conservation, and documentation, the marginal cost for holding accessions with the operational costs for maintenance of environment and equipment is taken for granted and therefore excluded from the fixed cost of physical capital or inflated/increased labor inputs. Besides the capital, labor, and operational costs in the total cost of conservation, several other factors are involved. In addition, a back-up facility for uninterrupted power supply and an off-site facility to perform special functions may be required for perpetuity.

Genebank operation is capital intensive, needing refrigerated modules and other equipment, and if this investment is expressed in annualized terms of the capital cost, it is very nominal at around $5 \%$. In operations, about $30 \%$ of the annual cost of the genebank operations could be attributed to labor costs, $65 \%$ to running or operational cost and energy requirements, and the remaining to the annualized cost of capital items. The lower labor costs compared with those reported by Pardey et al. [8] are because of cheaper labor wages in India, and because they included the costs of senior scientific and technical staff in the labor cost. In this study, such staff costs have been considered constant and not included in the labor cost. 
Implication and Scope

The above discussion clearly demonstrates that the costs of conservation under controlled $e x$ situ conditions are fairly high, and call for rationalization in use. For the same number of accessions and crops, the costs of conservation under medium-term storage (in modules maintaining temperatures between 4 and $10{ }^{\circ} \mathrm{C}$ and $\mathrm{RH}$ of $35-45 \%$ ) are higher than the costs of conservation under long-term storage (at $-18{ }^{\circ} \mathrm{C}$ ). From an economic standpoint and for consolidation of holdings, this study suggests restricting the number of accessions in MTS, and placing a majority of the accessions under long-term conditions. Active collections with no immediate potential value or use could be transferred to long-term stores under a black-box arrangement (small quantity, not to be opened until required for restoration, safety, and use). Second, as the establishment and running costs of MTS are higher, it would be appropriate to suggest that private/civil societies/NGOs and even public institutions could pool their resources for the conservation of active collections (regularly used in research and crop improvement) to reduce the costs further. This arrangement may particularly be advisable for tropical developing countries, where temperature and humidity fluctuations are high, needing greater energy for the maintenance of controlled conditions. Pooling may be both on a regional or crop basis, depending on the richness in crop diversity or importance of a crop to the region. For large countries like India, the establishment of national repositories on a regional or crop basis involving all stakeholders may further help in cost reduction and avoid duplication of efforts.

All costs have some marginal component that can be reduced by rationalization. For example, acquisition of new accessions can be rationalized by avoiding duplicates. If the seed material of accessions received is not of the recommended quality and insufficient in quantity, multiplication would increase the cost. Therefore, collection/ introduction of quality seed in sufficient quantities can reduce the cost. Regular monitoring and appropriate upkeep and multiplication in larger quantity during regeneration can further reduce the cost.

Seeds are maintained in medium-term storage to facilitate dissemination for use in research and crop improvement. As per international genebank standards, the monitoring is advised at 5-year intervals in the case of MTS, and 10-year intervals in the case of LTS. However, the monitoring results of this study reflect that most crops/ accessions are able to retain seed viability even after a gap of 10-15 years, suggesting that the interval period for monitoring could be extended [10]. Increased monitoring intervals will reduce the number of regeneration cycles, thus reducing the cost and chances of genetic alteration.
Further, the operation costs are nearly constant, irrespective of the crop and number of accessions stored. Therefore, operation of a genebank facility with storage to full capacity would bring down the costs substantially. In a multiple-crop genebank, the same store can be used to its full capacity irrespective of crops with diverse genetic constitution, thereby reducing the cost of conservation. Other options, such as safety-duplication of accessions in one location with black-box arrangement with quantity of seed sufficient for single regeneration, avoiding conservation of the same accessions in different national facilities, could help further reduce the costs.

In the present context of economic prudence, the cost of conservation with respect to capital costs is more sensitive to change in the rate of interest, than to change in the initial protocol or procurement. Lower rates of interest result in higher present value of these cost streams. But the interest cost, securing long-term commitment falls proportionally when the interest rates are lower. Regeneration costs constitute a significantly larger share in the overall cost of conservation for cross-pollinated and often cross-pollinated crops than for self-pollinated crops, so there would be corresponding larger cost consequences from change in initial regeneration cost in cross-pollinated and often crosspollinated than later if regeneration is deferred, specially with higher rates of interest.

If the conservation objective is achieved with one initial regeneration cycle, for 25-50 years, without further regeneration, the present value of commitment to conserve seed comes down further at a specific rate of interest. Such information could be used to calculate the benefits accrued from upgraded genebank activities/facilities and the resultant longer storage life of the seed. Larger multiplication would reduce the value of present average cost of accessions. These savings in the cost are marginal but bound with estimates of the benefit for improved seed storage. They do not account for the benefits derived from increased safety and with lesser chances of genetic drift or shift during regeneration and storage.

Conserving seed is a capital-intensive affair. In addition to the capital expenses, a sizeable investment is required in laboratories and earthquake-proof storage facilities. The costs are highly sensitive to several factors including the candidate crops themselves, breeding behavior, and seed size and composition, but primarily because of the cost of multiplication/regeneration under controlled pollination conditions and the comparatively larger population size required to capture the total genetic/allelic diversity of an accession. The other factors contributing to higher costs are low multiplication rate (groundnut), space required (castor), and the growth habit (perennial pigeonpea and cotton, larger canopy), which corroborates earlier observations [6]. Other cost variables include the cost of 
electricity, labor salary, cost of fuel and spares, maintenance of plant and equipment, particularly in the context of the continued high inflation rates around world.

Conservation is a global priority today, and there are several approaches available, including the most widely accepted ex situ mode. An attempt has been made in this study to examine some of the issues involved in costing $e x$ situ seed banks. The information brought together in this study could provide a framework for economic decisionmaking, in view of the different needs of diverse stakeholders (after due rationalization of the proposed costing structures to take into account the annual inflation rate and the predictable escalation in costs over the years). Be it community-based promotion and conservation of products of the agricultural heritage site/systems as envisaged by Singh and Varaprasad [11], or reliable decision-making support for national policy-makers confronted with the need to sanction new genebanks, or even research and development for various PGRFA storage options, this study could serve as a key reference point for the way forward.

Acknowledgments The authors gratefully acknowledge the funding support and facilities provided by the Indian Council of Agricultural Research (ICAR), New Delhi, India.

\section{References}

1. Business.mapsofindia.com Inflation Cached Inflation in India, 2011 Inflation Rates

2. Epperson JE, Pachico DH, Guevara CL (1997) A cost analysis of maintaining cassava plant genetic resources. Crop Sci 37:16411649

3. FAO (2010) The second report on the state of the world's plant genetic resources for food and agriculture. FAO, Rome
4. Jarret RL, Florkowski WJ (1990) In vitro active vs field genebank maintenance of sweet potato germplasm: major cost considerations. Hortic Sci 25:141-146

5. Koo B, Pardey PG, Wright B (2003) The price of conserving agricultural biodiversity. Nat Biotechnol 21:126-128

6. Koo B, Pardey PG, Wright B (2003) Conserving genetic resources for agriculture: counting the cost. In: Pardey PG, Koo B (eds) Research at glance, biotechnology and genetic resources policies. Brief 6. International Food Policy Research Institute, Washington, DC

7. Pardey PG, Koo B, Wright BD, Van Dusen ME, Skovmand B, Taba S (1999) Costing the ex situ conservation of genetic resources: maize and wheat at CIMMYT. DPTD Discussion Paper \#52

8. Pardey PG, Koo B, Wright BD, Van Dusen ME, Skovmand B, Taba S (2001) Costing the conservation of genetic resources: CIMMYT ex situ maize and wheat collections. Crop Sci 41:1286-1299

9. Pardey PG, Skomand B, Taba S, Van Dusen ME, Wright BD (1998) The cost of conserving maize and wheat genetic resources ex situ. In: Samle M (ed) Farmers, gene banks and crop breeding: economic analyses of diversity in wheat, maize and rice. Kluwer Academic Publishers, Boston

10. Singh AK (2007) Conventional conservation of agricultural and horticultural crops diversity. In: Singh AK, Srinivasan K, Saxena $\mathrm{S}$, Dhillon BS (eds) Hundred years of plant genetic resources management in India. NBPGR (ICAR), New Delhi, pp 191-210

11. Singh AK, Varaprasad KS (2008) Criteria for identification and assessment of agro-biodiversity heritage sites: evolving sustainable agriculture. Curr Sci 94(9):1131-1138

12. Walters C (2003) Optimizing seed banking procedures. In: Smith RD, Dickie JB, Lenington SH, Pritchard HW, Probart RJ (eds) Seed conservation: turning science into practice. Royal Botanic Gardens, London, pp 723-743

13. http://www.nbpgr.ernet.in/monthly_progress/cons_dec11.pdf. Accessed 31 Dec 2011

14. http://r4dreview.org/2010/09/to-conserve-or-not-to-conserve/. Accessed 28 Sep 2010 